\title{
MOLECULAR DISKS AROUND YOUNG STARS
}

\author{
Anneila I. Sargent, ${ }^{1}$ and Steven V. W. Beckwith, ${ }^{2}$ \\ ${ }^{1}$ California Institute of Technology, Pasadena, CA 91125 \\ ${ }^{2}$ Cornell University, Ithaca, NY 14853
}

\section{Introduction}

There is now a substantial body of evidence that disk-like structures of gas and dust surround many protostars and young stellar objects (e.g. Rodriguez, these proceedings). We are currently studying a number of these disks to establish whether they have properties compatible with those attributed to the proto-solar nebula, in the early stages of evolution of our solar system. The frequency with which such proto-planetary disks are associated with young stars is also being investigated.

With the 30-meter IRAM telescope, and in collaboration with R. Chini and R. Güsten of the MaxPlanck-Institut für Radioastronomie, Bonn, we have carried out a survey of $T$ Tauri stars in the TaurusAuriga cloud complex to ascertain the fraction that show $1.2 \mathrm{~mm}$ continuum emission. As discussed below, the detection of millimeter-wave emission from the environs of these stars implies the presence of gaseous circumstellar disks. Aperture synthesis observations of selected objects from this sample are under way at Owens Valley Radio Observatory (OVRO), using the Millimeter Wave interferometer; from the resulting maps the morphology and kinematic properties of individual disks can be determined.

\section{The Disk around HL Tauri}

Interferometric molecular line observations not only provide high spatial resolution information about the morphology and mass of the gas around young stars, but also permit studies of the velocity field. Because such measurements are insensitive to structures greater than about $30^{\prime \prime}$ in size, the circumstellar environments of objects which are embedded in much larger clouds can be investigated.

Aperture synthesis observations of HL Tauri, for example, in the $J=1 \rightarrow 0$ transitions of CO and ${ }^{13} \mathrm{CO}$, have demonstrated the presence of an elongated structure around this young $\mathrm{T}$ Tauri star (Beckwith et al. 1986; Sargent and Beckwith 1987). About $0.1 \mathrm{M}_{\odot}$ of gas appears to be moving in Keplerian orbits around an approximately $1 \mathrm{M}_{\odot}$ star. Even in the ${ }^{13} \mathrm{CO} \mathrm{J}=1 \rightarrow 0$ line the emission appears to be optically thick and can be detected as far as $2000 \mathrm{AU}$ from the star. Taken together, these properties suggest that here the very early stages of evolution of a planetary system like our own are being viewed. 
Since aperture synthesis mapping of systems such as HL Tauri is a time-consuming project, the selection of optimum candidates for interferometric observations is critical. Continuum emission from the disks under consideration is expected to be optically thin at millimeter wavelengths. Thus, observations at $1.2 \mathrm{~mm}$ should provide a direct measure of the amount of circumstellar material associated with stars. Other indicators of circumstellar disks, such as infrared spectral energy distributions (Adams, Lada and Shu 1987, 1988; Kenyon and Hartmann 1987; Bertout, Basri and Bouvier 1988) or infrared excesses (Strom et al. 1989; Cohen, Emerson and Beichman 1989), preferentially reflect disk temperatures. The magnitude of the $1.2 \mathrm{~mm}$ flux is thus a better discriminant of candidates for the relatively time-consuming, interferometric observations.

Our $1.2 \mathrm{~mm}$ continuum survey of the Taurus-Auriga cloud encompassed 87 young stars (Beckwith et al. 1989). The $\approx 10^{\prime \prime}$ resolution of the 30 meter IRAM telescope at $1.2 \mathrm{~mm}$ corresponds to a spatial scale of $1400 \mathrm{AU}$ at the Taurus distance, $140 \mathrm{pc}$, and discrimates against source confusion. The sample included both classical T Tauri (TT) and weak-line T Tauri stars (WTT). WTT's are defined as those having $\mathrm{H} \alpha$ equivalent widths of less than $10 \AA$ (c.f. Strom et al. 1989). Of the TT stars, 53\% showed $1.2 \mathrm{~mm}$ emission, while $29 \%$ of the WTT's were detected. Similar fractions of TT's are suggested by searches which rely on detections of infrared emission much in excess of that expected from the stellar photospheres (Strom et al. 1989), or on models of the infrared spectra (Cohen, Emerson, and Beichman 1989). For two stars, HL Tau and DG Tau, continuum emission at $1.4 \mathrm{~mm}$ has been measured independently at OVRO (Woody et al. 1989). Within the uncertainties, the fluxes agree very well with those measured at IRAM, $880 \mathrm{mJy}$ and $450 \mathrm{mJy}$ for HL Tau and DG Tau respectively. It is noteworthy that the proportion of detected WTT's is not very different from the fraction of TT's seen, refuting the hypothesis that $\mathrm{H} \alpha$ weakness implies a lack of circumstellar material (Walter 1987).

It is assumed that these $1.2 \mathrm{~mm}$ fluxes are due mainly to thermal emission from particles around the stars. A comparison of our results with radio fluxes measured at the VLA by Bieging, Cohen, amd Schwartz (1984) indicates little contribution from free-free emission. The $6 \mathrm{~cm}$ flux of DG Tau, for example, one of the strongest sources at cm wavelengths, suggests a $1.2 \mathrm{~mm}$ flux of at most $36 \mathrm{mJy}$, only $8 \%$ of the observed value. For thermal emission, the expected visual extinction, $A_{v}$, to each star can be calculated, assuming various particle distributions. In general, the $1.2 \mathrm{~mm}$ fluxes imply high values of $A_{V}$. However, the measured $A_{V} s$ are rarely greater than $3^{\mathrm{m}}$, indicating that the emitting particles must be distributed very non-uniformly, most probably in flattened disks. 
As discussed above, the $1.2 \mathrm{~mm}$ fluxes provide a good diagnostic for circumstellar disks suitable for interferometer mapping. With a flux of $440 \mathrm{mJy}$, DG Tau is, like HL Tau, one of the strong 1.2 mm sources. Also, [SII] observations of DG Tau (Mundt 1988) show a blue-shifted jet emanating from the star in the direction PA $226^{\circ}$, almost parallel to the magnetic field, as might be expected if the circumstellar mass distribution were anisotropic (see Hodapp 1984). Aperture synthesis mapping of DG Tau in the $\mathrm{J}=1 \rightarrow 0{ }^{13} \mathrm{CO}$ line at $110 \mathrm{GHz}$ was carried out at OVRO in 1988-89. Continuum observations at $2.7 \mathrm{~mm}$ were acquired simultaneously and show a $71 \mathrm{mJy}$ source, unresolved in the $6^{\prime \prime} \times 5^{\prime \prime}(800$ $\times 700 \mathrm{AU})$ beam. Following the arguments of Beckwith et al. (1986), with excitation temperature 50 $\mathrm{K}$ and dust emissivity directly dependent on frequency (see also Woody et al. 1989), this continuum measurement leads to a mass of approximately $0.05 \mathrm{M}_{\odot}$ for the circumstellar material.

A map of the ${ }^{13} \mathrm{CO}$ emission integrated over the velocity range 6.4 to $7.6 \mathrm{~km} \mathrm{~s}^{-1}$ is presented in Figure 1. No emission above a $2 \sigma$ level of $300 \mathrm{mJy} / \mathrm{beam}$ was detected outside this velocity range. An elongated feature of radius $2000 \mathrm{AU}$ is centered approximately on the star. The elongation is along PA

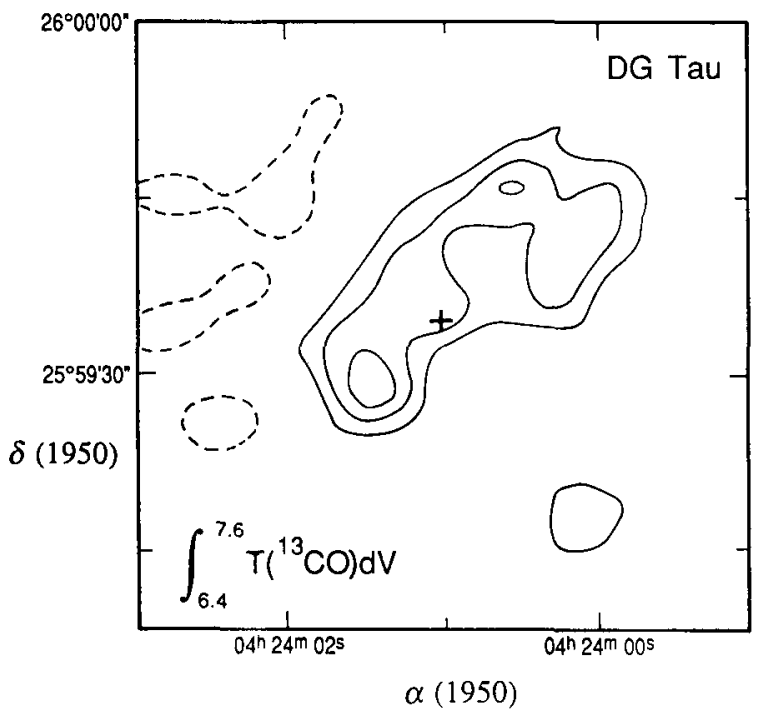

Figure 1. A map of the integrated ${ }^{13} \mathrm{CO}$ emission from DG Tau. Contours begin at $300 \mathrm{mJy} / \mathrm{beam}$, the $3 \sigma$ level, and are separated by $200 \mathrm{mJy}$. A cross marks the location of the $2.7 \mathrm{~mm}$ continuum source, which coincides with the stellar position. 
$130^{\circ}$, perpendicular to the optical jet detected by Mundt (1988). The total integrated flux, $6.2 \mathrm{Jy} \mathrm{km} \mathrm{s}^{-1}$, indicates a mass around $0.02 \mathrm{M}_{\odot}$ (c.f. Sargent and Beckwith 1987).

Maps of the ${ }^{13} \mathrm{CO}$ emission at $0.26 \mathrm{~km} \mathrm{~s}^{-1}$ resolution demonstrate that the position of the peak changes with velocity; above $7.0 \mathrm{~km} \mathrm{~s}^{-1}$ emission tends to be confined to the the south-east half of the elongated structure of Figure 1, while below $7 \mathrm{~km} \mathrm{~s}^{-1}$ emission is predominantly from the north-west region. For gravitational binding of this circumstellar gas, a stellar mass of $1.4 \mathrm{M}_{\odot}$ is required. Although uncertain, the mass of DG Tau inferred from its position on the HR diagram is very close to this value, $1.8 \mathrm{M}_{\odot}$, suggesting that the gas is bound to the star. Although the evidence for Keplerian rotation is not as compelling as in the case of HL Tau, the two systems are remarkably similar and it is likely that DG Tau is also surrounded by a proto-planetary disk.

\section{Discussion}

It seems reasonable to expect that the detectable mass in a circumstellar disk will diminish with time, the circumstellar environment becoming more diffuse and possibly accumulating into larger structures like planets. While estimates of the disk masses may be derived from continuum and line interferometry, as described above, the requisite observations exist for only a few sources. However, it has recently been shown that the mass of a circumstellar disk may be determined relatively accurately from only a few observed parameters (Beckwith et al. 1989). Briefly, the infrared spectral energy distribution shortward of about $100 \mu \mathrm{m}$, where emission is optically thick, specifies the temperature distribution in the disk; knowing this, and the strength of the millimeter flux (where emission is predominantly optically thin), the disk mass may be calculated.

This method has been applied to all of our $1.2 \mathrm{~mm}$ sources for which infrared fluxes are available. We find disk masses ranging between $0.001 \mathrm{M}_{\odot}$ and about $0.5 \mathrm{M}_{\odot}$. Average disk temperatures range from a few $\times 10 \mathrm{~K}$ to a few $\times 100 \mathrm{~K}$. The sensitivity was such that most systems with masses comparable to that of the proto-solar nebula, $0.01 \mathrm{M}_{\odot}$, should have been deteceded. Calculations of the spectral energy distribution of HL Tau fit well with the observations for a disk mass of $0.09 \mathrm{M}_{\odot}$. Likewise, DG Tau requires a disk mass of $0.03 \mathrm{M}_{\odot}$. Both values are well within the ranges suggested by the interferometer measurements.

Comparisons of the disk masses and temperatures with the ages of the stars (based on their positions with respect to isochrones in temperature-luminosity plots) indicate no correlation between mass and age. Over a time-scale of $10^{5}$ to $10^{7}$ years there is no evidence of the mass in the disks decreasing. There is a very slight tendency for the disks to become cooler with age. Strom et al. 1989 have suggested on the 
basis of IRAS flux measurements that disk masses do show some signs of evolution over this time span; their results, deriving from the optically thick infrared regime may reflect decreasing temperatures rather than decreasing mass.

In summary, we have found that approximately $50 \%$ of a sample of solar-type, pre-main sequence stars have circumstellar disks. There is little evidence of mass evolution for stars with ages between $10^{5}$ and $10^{7}$ years, although older disks could be cooler. Aperture synthesis mapping of two of these stars has confirmed the presence of disk-like structures around them. The masses, radii and velocity patterns observed are consistent with those of the proto-solar nebula, suggesting that planetary systems may be forming around some of these objects.

\section{References}

Adams, F. C., Lada, C. J., and Shu, F. H. 1987, Ap. J., 312, 788.

Adams, F. C., Lada, C. J., and Shu, F. H. 1988, Ap. J., 326, 865.

Beckwith, S. Sargent, A. I., Chini, R. and Gusten, R. 1989, Ap. J., submitted.

Beckwith, S., Sargent, A. I., Scoville, N. Z., Masson, C. R., Zuckerman, B., and Phillips, T. G. 1986, Ap. J., 309, 755.

Bertout, C., Basri, G., and Bouvier, J. 1988 Ap. J., 330, 350.

Cohen, M., Emerson, J. P., and Beichman, C. A. 1989, Ap. J., in press.

Hodapp, K.-W. 1984, Astr. Ap., 141, 255.

Kenyon, S. J., and Hartmann, L. 1987, Ap. J., 323, 714.

Mundt, R. 1988, in NATO Advanced Study Institute "Formation and Evolution of Low Mass Stars", ed. A. K. Dupree and M. T. V. T. Lago.

Sargent, A. I., and Beckwith, S. 1987, Ap. J., 323, 294.

Strom, K. M., Strom, S. E. Edwards, S., Cabrit, S., and Skrutskie, M. F. 1989, A. J., 97, 1451.

Walter, F. M. 1987, Pub. A. S. P., 99, 31.

Woody, D. P., Scott, S. L., Scoville, N. Z., Mundy, L. G., Sargent, A. I., Padin, S., Tinney, C. G., and Wilson, C. D. 1989, Ap. J. (Letters), 337, L41.

\section{Discussion:}

ELSÄSSER: Is there any correlation between the orientation of the disks you resolved, projected on the sky?

SARGENT: I presume you are referring to their orientations not just relative to one another, but also to the local magnetic field. In fact, the three disks we have examined in the Taurus cloud, HL TAU, L1551 IRS5 and DG TAU are all similary oriented, close to PA 135, which is perpendicular to the local magnetic field direction.

LARSON: I would guess that the inner parts of protostellar disks evolve faster than the outer parts, and that your data probe mainly the outer parts. What radii do your results typically refer to? 
SARGENT: It is true that at present we are investigating large radii. The disks I have shown have radii of order $1000 \mathrm{AU}$. The smallest disk we have detected in molecular gas, L1551 IRS5, has a radius around $500 \mathrm{AU}$. But of course the continuum sources, which are mostly unresolved in 6 " beams, show that the bulk of the material is confined to regions of radii $\sim 500 \mathrm{AU}$. With higher resolution, especially at $1 \mathrm{~mm}$, we should be able to probe a little deeper.

SCHULZ: The "usual" dust emission $\beta$ is about 2 . Could you comment on your value of 1 ?

SARGENT: For most of our sources we can fit the $1.3 \mathrm{~mm}$ and $2.6 \mathrm{~mm}$ points in the spectral energy distributions only by adopting $\beta=1$. A study of the $1.4 \mathrm{~mm}$ emission from a variety of galactic sources carried out at Owens Valley Radio Observatory (Woody et al.,1989) also suggested $\beta=1$ when comparison with the $2.6 \mathrm{~mm}$ fluxes was made. Although $\beta=2$ is generally expected for both crystalline and amorphous materials (Tielens and Allamandola,1987), $\beta=1$ can result for layared materials where phonons are limited in two dimensions. Values of 1 and 1.5 have been found for amorphous carbon and layered silicates (Koike, Hasegawa and Manabe, 1980; Day, 1976).

CAMERON: Do you have a feeling for how far out into the far-IR/ submillimetre wavelength regime you have to go before the continuum emission from the disks you have observed becomes optically thin?

SARGENT: Our data on HL TAU and DG TAU show that they are still optically thick at $100 \mu \mathrm{m}$ and thin by $1 \mathrm{~mm}$. We have no observations at intermediate wavelengths, although we expect to acquire these at the Caltech Submillimeter Observatory next season.

ZINNECKER: Your result that about $50 \%$ of the T Tauri stars are detected at $1 \mathrm{~mm}$ (indicative of cool dusty circumstellar disks) leads me to ask about the other $50 \%$ that you did not detect: could those be binary stars (whose incidence is of the order of $50 \%$ )? Did you detect continuum emission from $\mathrm{T}$ Tauri binary stars (e.g., T Tau)?

SARGENT: Yes, we did detect $1.3 \mathrm{~mm}$ continuum emission from T Tauri. Weintraub et al. (1989) have in fact argued for a circumbinary disk on the basis of OVRO measures. I doubt if the presence of a companion would prevent there being a disk. Recent speckle measures of $\mathrm{Z} \mathrm{CMa}$ also appear to show a close companion and a disk (Koresko, Beckwith and Sargent 1989). What I find quite interesting is that Becklin and Zuckerman (1988), in their studies of Vega-like objects, also find a $50 \%$ detection rate for systems with circumstellar structure. 